\title{
Gallium Ga 68-labeled GRPR Antagonist BAY86-7548
}

National Cancer Institute

\section{Source}

National Cancer Institute. Gallium Ga 68-labeled GRPR Antagonist BAY86-7548. NCI

Thesaurus. Code C121773.

A radioconjug ate containing a synthetic bombesin receptor antagonist targ eting the gastrin-releasing peptide receptor (GRPR), that is linked by the macrocyclic chelator 1,4,7,10-tetraazacyclododecane-1,4,7,10-tetraacetic acid (DOT A) to the radionuclide gallium $(\mathrm{Ga}) 68$, with potential use in diagnostic imaging using positron emission tomography/computed tomography (PET/CT). Upon administration of gallium Ga 68 GRPR antagonist BAY86-7548, the peptide moiety targets and binds to GRPR. Upon PET/CT, GRPR-expressing tumor cells can then be visualized. GRPR, also called bombesin receptor 2 (BB2), is a G protein-coupled seven-transmembrane receptor belong ing to the bombesin receptor family. It is overexpressed in certain types of cancers. 